\title{
Towards ultrasound enhanced mid-IR spectroscopy for sensing bacteria in aqueous solutions
}

\author{
Stephan Freitag, Andreas Schwaighofer, Stefan Radel, Bernhard Lendl* \\ Institute of Chemical Technologies and Analytics, Technische Universität Wien, \\ Getreidemarkt 9/164, A 1060 Vienna, Austria
}

Copyright 2018 Society of Photo-Optical Instrumentation Engineers (SPIE). One print or electronic copy may be made for personal use only. Systematic reproduction and distribution, duplication of any material in this publication for a fee or for commercial purposes, and modification of the contents of the publication are prohibited. The published version of this accepted manuscript is available under: S Freitag, A Schwaighofer, S Radel, B Lendl, Towards ultrasound enhanced midIR spectroscopy for sensing bacteria in aqueous solutions Proc. SPIE 10491, Microfluidics, BioMEMS, and Medical Microsystems XVI, 2018. DOI: 10.1117/12.2290390.

\begin{abstract}
We employ attenuated total reflection (ATR) mid-IR technology for sensing of bacteria present in aqueous solution. In ATR spectroscopy, the penetration depth of the evanescent field extends to approx. 1-2 micrometers into the aqueous solution depending on the refractive index of the employed materials ( $\mathrm{Si}, \mathrm{ZnS}, \mathrm{Ge}$ ) used as attenuated total reflection (ATR) element and the geometry of the optical set-up. Due to the flow profile in the microfluidic cell, an additional force is required to bring particles into the evanescent field for measurement. For that purpose, we employ standing ultrasound waves produced between a sound source vibrating at approx. $2 \mathrm{MHz}$ and the ATR crystal acting as a reflector. This ultrasonic trap is integrated into the microfluidic channel. As aqueous solution is passing through that acoustofluidic cell, particles are concentrated in the nodal plane of the standing ultrasound wave, forming particle conglomerates. By selecting appropriate experimental conditions, it is then possible to press bacteria against the crystal surface for interaction with the evanescent wave (as well as to keep them away from the ATR element). Our current work aims at establishing a custommade US-ATR-IR setup for signal enhancement of bacteria (e.g. E. coli, P. aeruginosa as well as Salmonella) in drinking water.
\end{abstract}

Keywords: mid-infrared, attenuated total reflection spectroscopy, ultrasound enhancement, FT-IR, E. coli

\section{INTRODUCTION}

Infrared (IR) spectroscopy is an established technique for the analysis of biological samples. It provides qualitative and quantitative information in a non-destructive and label-free manner, because inherent vibrations of molecular vibrations are detected. ${ }^{1}$ In the food and pharmaceutical industries, IR spectroscopy is applied in analysis of microorganisms for rapid identification and screening of life-threatening pathogens, biotechnological process control and microbiological quality control. ${ }^{2}$ For measurements in water, which is a strong absorber in the mid-IR region and allows merely interaction lengths of a few tens of micrometers, attenuated total reflection (ATR) configurations are the most commonly employed. Using this technique, the light is totally reflected within the optically denser ATR element, forming an evanescent field that interacts with the sample, with typical penetration depths of approximately 1 to $2 \mu \mathrm{m} .{ }^{3}$ In order to force microscopic analyte particles into the evanescent field of an ATR probe, ultrasound (US) manipulation has been successfully employed. ${ }^{4-6}$ When US is imposed on a liquid containing particles e.g.: microorganisms, the latter will be affected by the acoustic radiation force. This effect is also called acoustophoresis. ${ }^{7}$ US acoustics proofed to be well-suited for use in microfluidics, because ultrasound frequencies of approx. $2 \mathrm{MHz}$ combined with the speed of sound in water $\left(1.5 \times 10^{3} \mathrm{~m} / \mathrm{s}\right) \mathrm{result}$ in a corresponding wavelength of $<1 \mathrm{~mm}$, which optimally fits for sub-millimeter sized microfluidic systems. ${ }^{8}$ When an ultrasound wave created by a piezo ceramic driven transducer is reversed by a reflector, interference occurs resulting in an US standing wave. Acoustic-fluidic microfluidics or acoustofluidics have shown potential in biomedical analytic tasks like cell separation, ${ }^{9}$ bead-based bioassays ${ }^{10}$ and concentration of bacteria in water. ${ }^{11}$ In combination with ATR-IR spectroscopy, acoustophoresis has been employed for in-line analysis of microorganisms i.e. yeast during fermentation. ${ }^{6}$ In this work, US-enhanced ATR-IR spectroscopy is presented for analysis of bacteria in water. Freshwater is a finite 
resource, essential for agriculture, industry and even human existence. ${ }^{12}$ Therefore, effective and sensitive monitoring of microbiological pathogens is needed to evaluate the presence of waterborne diseases or contamination. Different types of bacteria can be present in water sources. For example, Escherichia coli $(E$. coli) is a good indicator for fecal contamination of drinking water. ${ }^{13}$ Salmonella infection causes 155.000 deaths per year in developed and non-developed countries and is therefore a persistent global burden. ${ }^{14}$ Further, Pseudomonas aeruginosa as a ubiquitous environmental bacterium can be found literally in every naturally occurring waterbody and is known for its resistance against disinfection. As a potent pathogen which can infect most devitalized tissues, it is a huge threat in hospital environments. ${ }^{15}$

A classic method for identification of bacteria is the cultivation approach. Therefore, the bacterium of interest is cultivated on different media containing e.g. $\mathrm{pH}$ indicator or chromophores to evaluate the metabolic and morphological properties of the target strain. ${ }^{16}$ Modern approaches in microbiology are based on molecular biological methods like polymerase chain reaction (PCR), nucleic acid sequencing, and deoxyribonucleic acid arrays. ${ }^{17}$ Because prior to testing, incubation steps of 18-24 $\mathrm{h}$ are needed, ${ }^{18}$ these methods are often time and/or labour consuming.

In this regard, US-enhanced ATR-IR spectroscopy poses an interesting alternative, because the standing ultrasonic wave can be used in a first step for accumulation of the bacteria in the sample cell. Subsequently, the enriched bacteria solution is forced towards the ATR element for interaction with the evanescent wave.

In this paper, we demonstrate the combination of an ATR-IR setup with US acoustics for measurements of bacteria in water.

\section{THEORETICAL BACKGROUND: ULTRASOUND ACOUSTICS}

The first description of the acoustic forces exerted on particles in suspension was performed by King in $1934 .{ }^{19}$ In this approach, a rigid sphere surrounded by an ideal fluid of zero viscosity was assumed, which was exposed to a plane ultrasonic standing wave. These considerations resulted in a non-vanishing axial force on the particles induced by the acoustic radiation pressure. The assumptions of King were extended to a compressible sphere by Yosioka and Kawasima, ${ }^{20}$ and later also the influence of the hosting fluid was included.

The primary radiation force $F_{z}^{r a d}$ has the major contribution to acoustic manipulation of spherical particles with the radius a, when it is small compared to the wavelength of the applied ultrasound wave.

$$
\begin{gathered}
F_{z}^{r a d}=4 \pi \cdot \phi(\tilde{\kappa}, \tilde{\rho}) \cdot(k a)^{3} \cdot E_{a c} \cdot \sin (2 k z) \\
\phi(\tilde{\kappa}, \tilde{\rho})=\frac{1}{3}\left(\frac{5 \tilde{\rho}-2}{2 \tilde{\rho}+1}-\tilde{\kappa}\right) \\
E_{a c}=\frac{p_{a}^{2}}{4 \rho_{0} c_{0}^{2}}
\end{gathered}
$$

Here, $\phi(\tilde{\kappa}, \tilde{\rho})$ denotes the acoustic contrast and $E_{a c}$ is the acoustic energy. The term $\sin (2 k z)$ reflects the periodical character of the force and expresses the half-wavelength pattern of the nodes generated by an ultrasound standing wave. It can be derived that the particle radius a and the wavelength $\mathrm{k}$ have a disproportionally high influence on the primary radiation force. Further, $F_{z}^{r a d}$ scales directly with the acoustic contrast, which describes the direction of the primary radiation force and can be either positive or negative. This term scales with the ratio in mass density $\left(\tilde{\rho}=\frac{\rho_{p}}{\rho_{M}}\right)$ and compressibility $\left(\tilde{\kappa}=\frac{\kappa_{p}}{\kappa_{M}}\right)$ between the particles $\mathrm{P}$ and the hosting medium $\mathrm{M}$. The speed of sound $\mathrm{c}$ in the medium and the mass density are connected with the compressibility by $\kappa=\frac{1}{\rho \cdot c^{2}}$. As shown in Equation 3, the magnitude of the acoustic energy transmitted into the medium is derived from the acoustic pressure amplitude $\left(p_{a}\right)$, the density $\left(\rho_{0}\right)$ and speed of sound $\mathrm{c}_{0}$. 


\section{EXPERIMENTAL}

FT-IR absorption measurements were performed using a Bruker Tensor 27 FT-IR spectrometer (Ettlingen, Germany) equipped with a liquid nitrogen cooled $\mathrm{HgCdTe}$ (Mercury Cadmium Telluride) detector. In the sample compartment of the spectrometer, a custom-made ATR setup was incorporated (depicted in Figure 1). ZnS was chosen as material for the ATR crystal due to its refraction index of 2.35 and VIS transparency, which enables the possibility to monitor US experiments

A

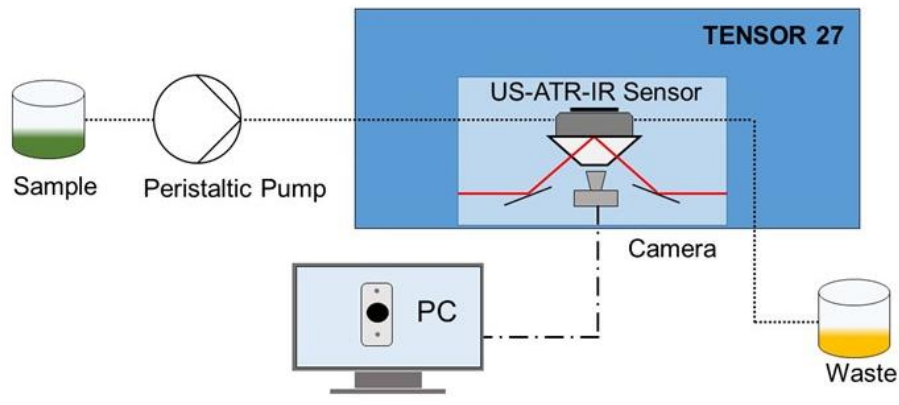

B

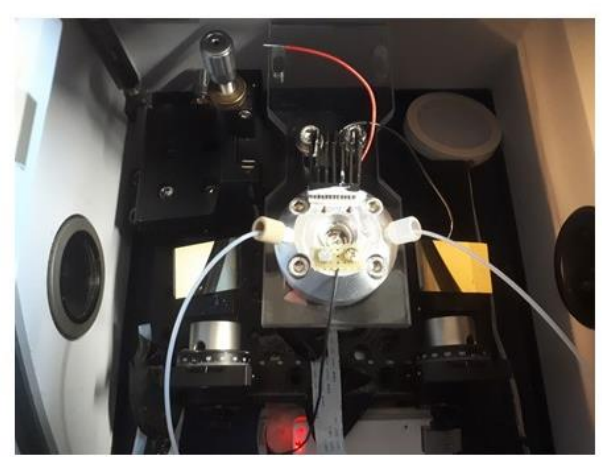

Figure 1: (A) Schematic of the experimental setup. (B) Picture of the custom-made ATR setup incorporated in the sample compartment of the Tensor 27 FT-IR spectrometer.

by a conventional VIS camera simultaneously to performing IR measurements. The IR beam was coupled into the ZnS crystal at an angle of incidence of $60^{\circ}$, allowing a single reflection in the ATR mode. A custom-made liquid-handling cell made of aluminum equipped with an ultrasound composite transducer consisting of a $10 \mathrm{~mm}$ PZT disc (lead zirconium titante, type PIC 181, PI Ceramics, Lederhose, Germany) with wraparound silver electrodes glued to a Macor cylinder with a two-component epoxy resin (Polytec EP 630, Polytec PT GmbH, Karlsbad, Germany). A custom-made driver was used to operate the US transducer, with a driving signal of approximately $1 \mathrm{~W}$ electrical power input. The operating frequency was adjusted to approx. 2.6 MHz. A Raspberry Pi single-board computer connected with a Raspberry Pi camera was mounted below the ATR crystal and served as tool to monitor particle enrichment throughout US experiments.

During measurements, the sample compartment was flushed with dry air for at least 15 minutes prior to spectrum acquisition. Spectra were acquired with a spectral resolution of $8 \mathrm{~cm}^{-1}$ in double-sided/forward-backward acquisition mode. A total of 128 scans were averaged per spectrum (acquisition time: $32.5 \mathrm{~s}$ ), which was calculated using a BlackmanHarris 3-term apodization function and a zero filling factor of 2 . All spectra were acquired at $25{ }^{\circ} \mathrm{C}$. Spectra were analyzed using the software package OPUS 7.5 (Bruker, Ettlingen, Germany). Savitzky-Golay smoothing (17 point) was applied and if necessary, absorption bands of water vapor in the atmosphere were subtracted. For all measurements, a spectrum of ultrapure water was recorded as reference. Bacteria experiments were performed with a non-pathogenic E. coli strain. Suspensions of $E$. coli were prepared by resuspending a bacteria pellet in ultrapure water. Ultrapure water (18 M $\Omega$ ) was used for the preparation of all solutions, obtained with a Milli-Q water purification system from Millipore (Bedford, USA). The bacteria were provided by the group of Dr. Antonio Varriale from Consiglio Nazionale delle Ricerche, Avellino, Italy.

\section{RESULTS AND DISCUSSION}

\subsection{ATR-IR spectra of $E$. coli}

An initial E. coli spectrum was recorded with the presented custom-made ATR-IR setup by pipetting aqueous suspension of $E$. coli $(38 \mathrm{~g} / \mathrm{l})$ on the ATR element. The IR spectrum depicted in Figure 2 was recorded after a settling time of 1 hour and showed the typical mid-IR features associated with bacteria. The amide I $\left(\sim 1650 \mathrm{~cm}^{-1}\right)$ and amide II $\left(\sim 1550 \mathrm{~cm}^{-1}\right)$ regions as well as the symmetric stretching vibrations of $\mathrm{COO}^{-}\left(1400 \mathrm{~cm}^{-1}\right)$ are typical protein absorption bands. Lipids can be linked to the symmetric $\mathrm{CH}_{2}$ bending vibration $\left(\sim 1450 \mathrm{~cm}^{-1}\right)$. Nucleic acids containing phosphate esters are represented through the stretching vibrations of phosphate $\left(1200-1300 \mathrm{~cm}^{-1}\right)$. Symmetric stretching vibrations of the $\mathrm{CO}-\mathrm{O}-\mathrm{C}$ group are characteristic for carbohydrates and occur at $1000-1100 \mathrm{~cm}^{-1} \cdot{ }^{21}$ 


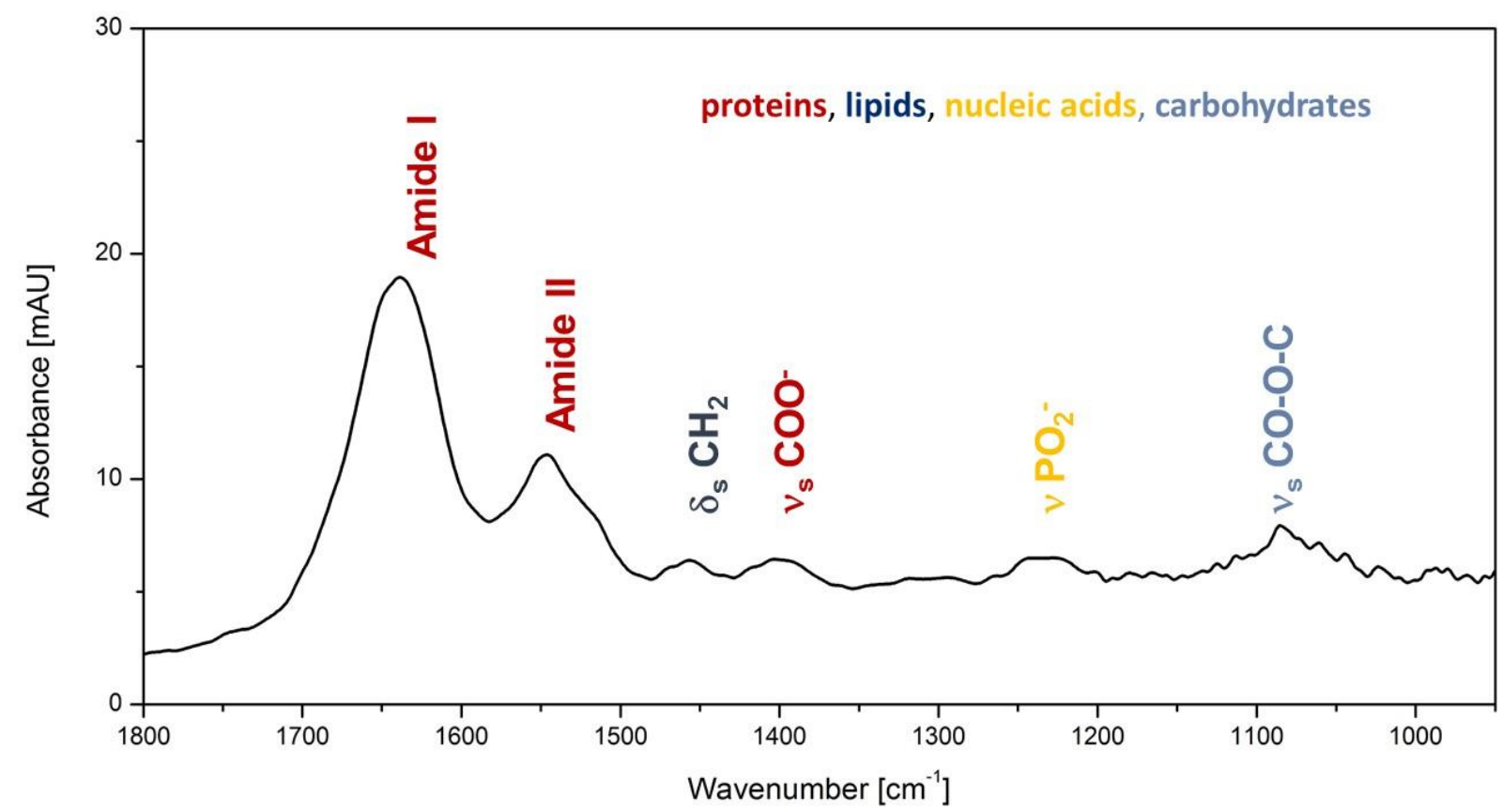

Figure 2: ATR-IR absorption spectrum of aqueous E. coli solution ( $38 \mathrm{~g} / \mathrm{l})$ after 1 hour of sedimentation on the ATR element.

\subsection{Ultrasound enhancement of ATR-IR spectra of $E$. coli}

IR measurements of E. coli with the US-ATR-IR sensor were performed after pumping the bacteria suspension into the ultrasound cell using a peristaltic pump. Throughout the entire experiment time, the cell was observed by the VIS camera. The pictures in Figure 3 show the US-ATR-IR cell before US activation and after 15 minutes of US exposure. The formation of E. coli conglomerates within the cell in the area below the US transducer is clearly identifiable (see Figure 4).

After that the cell was filled with $E$. coli suspension $(5 \mathrm{~g} / \mathrm{l})$, the pump was stopped and the US transducer was turned on for 12 minutes. At this point, the recorded IR spectrum does not show any IR features characteristic for bacteria (black solid line in Figure 4). In the next step, the US transducer was turned off, and the bacteria were allowed to settle. After 12 minutes of sedimentation time, an IR spectrum was recorded featuring the typical absorption bands of bacteria (compare to Figure 2), indicating the presence of $E$. coli in the evanescent field (red solid line). Subsequently, the US transducer was

A Ultrasound off

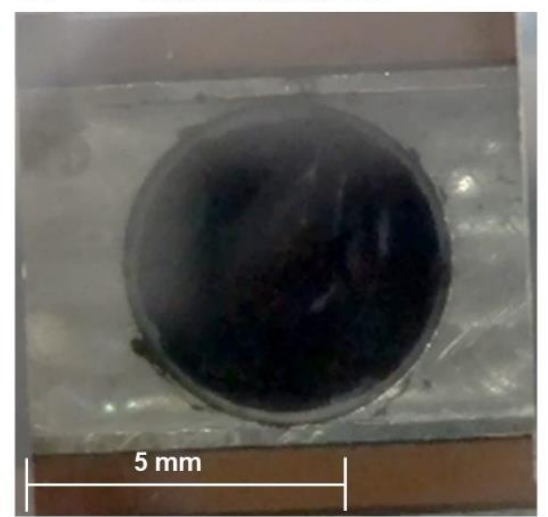

B Ultrasound on

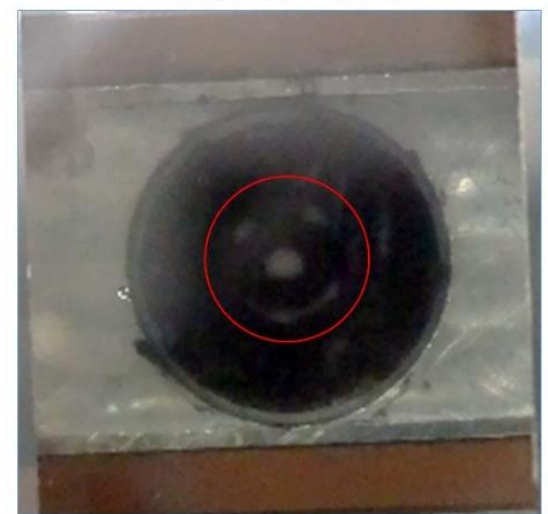

Figure 3: Pictures of the US cell with ultrasound transducer turned (A) off and (B) on. Formation of the E. coli conglomerates below the US transducer is highlighted by the red circle. 
reactivated and an IR spectrum was recorded, which did not show typical bacteria IR bands (green dashed-dotted line). After turning off the US transducer again, followed by 12 minutes of sedimentation time, the recorded IR spectrum depicts characteristic IR features of bacteria again (blue dashed-dotted line). This recorded data indicates that upon turning on the US transducer, the ultrasonic wave captures the proteins within the cell below the transducer element. Apparently, at the applied US frequency the nodal planes of the ultrasound standing wave lie outside the evanescent wave. Upon allowing some sedimentation time, the bacteria settle down within the range of the evanescent wave. Future work will focus on optimizing the frequency settings and cell dimensions to allow for deliberate manipulation of the nodal plane position.

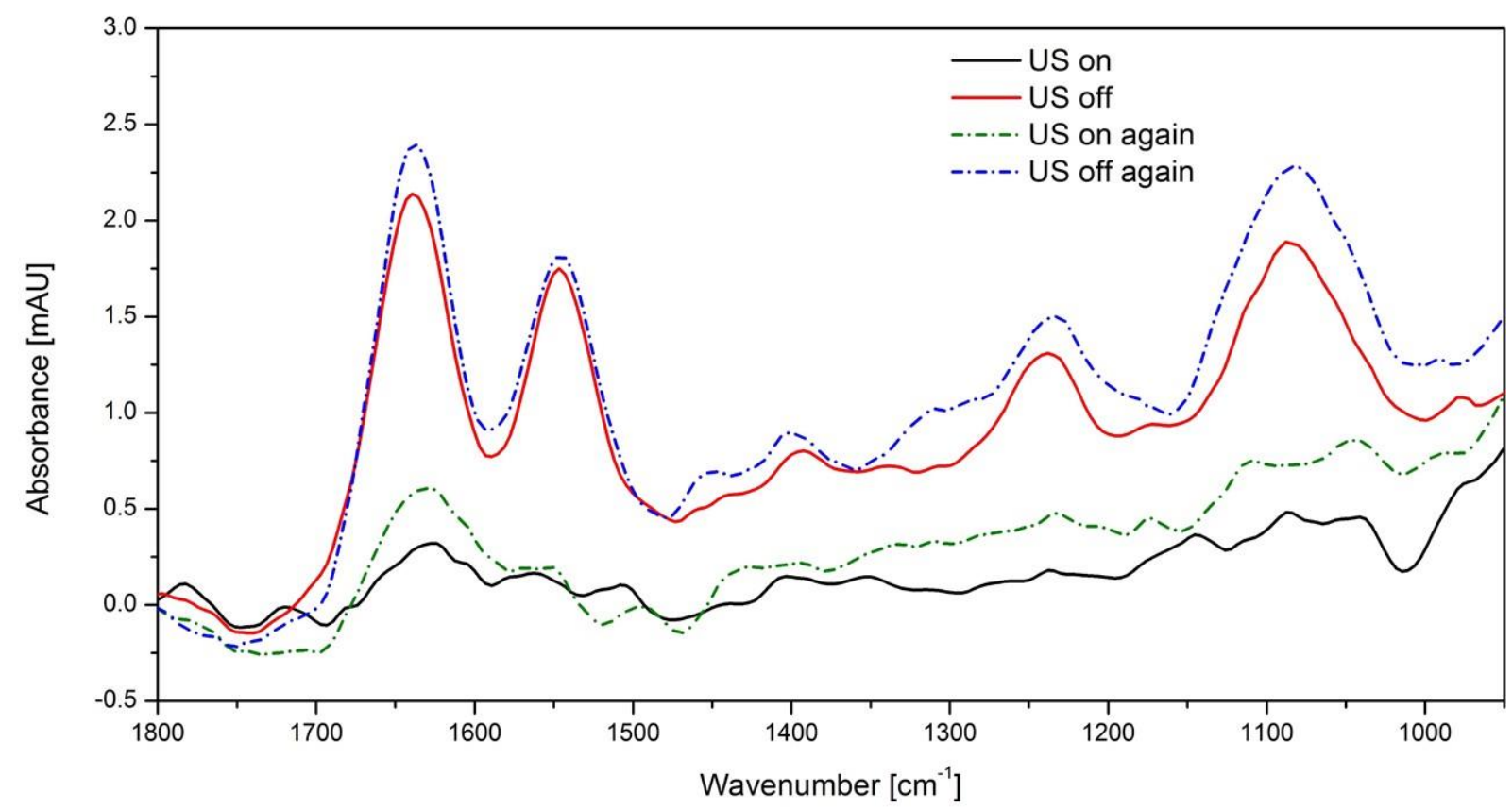

Figure 4: Infrared spectra of E. coli solution $(5 \mathrm{~g} / \mathrm{l})$ with and without continued exposure to ultrasound. E. coli solution after 12 minutes of ultrasound exposure (black solid line), E. coli solution after 12 minutes of ultrasound exposure followed by 12 minutes of settling time without ultrasound exposure (red solid line), E. coli solution after further exposure to ultrasound for 12 minutes (green dashed-dotted line), E. coli solution after 12 minutes of settling time without ultrasound exposure (blue dasheddotted line).

\section{CONCLUSION AND OUTLOOK}

In this study, we presented results of the ongoing research towards ultrasound enhanced mid-IR spectroscopy of bacteria in aqueous solutions. It was demonstrated that our custom-made US-ATR-IR sensor is capable of acquiring IR spectra of E. coli. By applying ultrasound, it was possible to trap E. coli with US and release them into the evanescent field resulting in spectra featuring the typical bacteria IR bands.

Our future efforts will focus on further miniaturization of the employed ultrasound cell as well as on the development of a rugged ATR setup. Furthermore, experiments at lower concentrations with bacteria of different kind, the use of quantum cascade lasers as IR radiation source and flow-through investigations are planned.

\section{ACKNOWLEDGEMENTS}

The authors thank our partners from Consiglio Nazionale delle Ricerche, Avellino Italy for providing E. coli bacteria pellets used in this study. This work has received funding from the European Union's Horizon 2020 research and innovation programme under the grant agreement no.: 731778 . 


\section{REFERENCES}

[1] Steele, D. "Infrared Spectroscopy: Theory", [Handbook of Vibrational Spectroscopy]. John Wiley \& Sons, Ltd, Hoboken, NJ, USA, (2006).

[2] Naumann, D. "Infrared spectroscopy in microbiology", [Encyclopedia of analytical chemistry]. John Wiley \& Sons, Ltd, Hoboken, NJ, USA, (2000).

[3] Griffiths, P. R. and De Haseth, J. A., [Fourier Transform Infrared Spectrometry], John Wiley \& Sons, Inc., Hoboken, NJ, USA, (2006).

[4] Radel, S., Brandstetter, M. and Lendl, B., "Observation of particles manipulated by ultrasound in close proximity to a cone-shaped infrared spectroscopy probe," Ultrasonics, 50(2), 240-246 (2010).

[5] Koch, C., Brandstetter, M., Lendl, B. and Radel, S., "Ultrasonic Manipulation of Yeast Cells in Suspension for Absorption Spectroscopy with an Immersible Mid-Infrared Fiberoptic Probe," Ultrasound Med. Biol., 39(6), 1094-1101 (2013).

[6] Koch, C., Brandstetter, M., Wechselberger, P., Lorantfy, B., Plata, M. R., Radel, S., Herwig, C. and Lendl, B., "Ultrasound-Enhanced Attenuated Total Reflection Mid-infrared Spectroscopy In-Line Probe: Acquisition of Cell Spectra in a Bioreactor," Anal. Chem., 87(4), 2314-2320 (2015).

[7] Bruus, H. "Acoustic Radiation Force on Small Particles", [Microscale Acoustofluidics]. Royal Society of Chemistry, (2014).

[8] Bruus, H. "Perturbation Theory and Ultrasound Resonances", [Microscale Acoustofluidics]. Royal Society of Chemistry, (2014).

[9] Barani, A., Paktinat, H., Janmaleki, M., Mohammadi, A., Mosaddegh, P., Fadaei-Tehrani, A. and Sanati-Nezhad, A., "Microfluidic integrated acoustic waving for manipulation of cells and molecules," Biosens. Bioelectron. 85, 714-725 (2016).

[10] Wiklund, M., Radel, S. and Hawkes, J. "Ultrasound-Enhanced Immunoassays and Particle Sensors", [Microscale Acoustofluidics]. Royal Society of Chemistry, (2014).

[11] Carugo, D., Octon, T., Messaoudi, W., Fisher, A. L., Carboni, M., Harris, N. R., Hill, M. and Glynne-Jones, P., "A thin-reflector microfluidic resonator for continuous-flow concentration of microorganisms: a new approach to water quality analysis using acoustofluidics," Lab Chip, 14(19), 3830-3842 (2014).

[12] Bartram, J. and Ballance, R., [Water quality monitoring: a practical guide to the design and implementation of freshwater quality studies and monitoring programmes], CRC Press, (1996).

[13] Edberg, S., Rice, E., Karlin, R. and Allen, M., "Escherichia coli: the best biological drinking water indicator for public health protection," J. Appl. Microbiol., 88(S1), 106S-116S (2000).

[14] Majowicz, S. E., Musto, J., Scallan, E., Angulo, F. J., Kirk, M., O'brien, S. J., Jones, T. F., Fazil, A., Hoekstra, R. M. and Studies, I. C. O. E. D. B. O. I., "The global burden of nontyphoidal Salmonella gastroenteritis," Clin. Infect. Dis. 50(6), 882-889 (2010).

[15] Mena, K. D. and Gerba, C. P. "Risk assessment of Pseudomonas aeruginosa in water", [Reviews of Environmental Contamination and Toxicology Vol 201]. Springer, (2009).

[16] O'hara, C. M., Weinstein, M. P. and Miller, J. M. "Manual and automated systems for detection and identification of microorganisms", [Manual of clinical microbiology]. American Society for Microbiology Press, Washington, D.C., USA, (2003).

[17] Nolte, F. and Caliendo, A. "Molecular detection and identification of microorganisms", [Manual of clinical microbiology]. American Society for Microbiology Press, Washington, D.C., USA, (2003).

[18] Kimberle, C. "Reagent, stains, and media: Bacteriology", [Manual of clinical microbiology]. American Society for Microbiology Press, Washington, D.C., USA, (2003).

[19] King, L. V., "On the acoustic radiation pressure on spheres," Proc. Royal Soc. A, 147(861), 212-240 (1934).

[20] Yosioka, K. and Kawasima, Y., "Acoustic radiation pressure on a compressible sphere," Acta Acust. Acust.,, 5(3), 167-173 (1955).

[21] Baker, M. J., Trevisan, J., Bassan, P., Bhargava, R., Butler, H. J., Dorling, K. M., Fielden, P. R., Fogarty, S. W., Fullwood, N. J. and Heys, K. A., "Using Fourier transform IR spectroscopy to analyze biological materials," Nat. Protocols 9(8), 1771-1791 (2014). 